\title{
Antibacterial activity of curcumin against Lebanese clinical isolates of Staphylococcus aureus
}

\begin{abstract}
The speed of emergence of resistance to newly introduced antimicrobial agents, by strains of common pathogens, has necessitated the search for newer medications. The use of medicinal plants and their extracts has become popular and promising. The methanol extract from Curcuma longa, a plant that has long been used by villagers, who noted its therapeutic properties, was tested, in this study, for its antibacterial activity against local, multiresistant strains of Staphylococcus aureus. The disc agar and well agar diffusion methods showed that the plant extract was able to inhibit the growth of the tested strains at different concentrations, revealing the genetic diversity of these strains. The emergence of resistant strains to the extract during the experiments, demonstrated the "drug fast" characteristic of the organism. Further studies are needed to conclude whether an active compound in the extract can be used in developing a new antibacterial agent.
\end{abstract}

Keywords: antibacterial effect, curcumin, S. aureus, medicinal plants, methanol extraction
Volume 4 Issue 2 - 2018

\author{
Patrick Abou Raji El Feghali, Riwa Ibrahim, \\ Tarek Nawas \\ Department of Natural Sciences, Lebanese American University, \\ Lebanon \\ Correspondence: Tarek Nawas, Department of Natural \\ Sciences, School of Arts and Sciences, Lebanese American \\ University, Beirut, Lebanon, Tel +96II 8676 I8, \\ Email tnawas@lau.edu.lb
}

Received: March 30, 2018| Published: April 10, 2018

\section{Introduction}

Antimicrobial agents are some of the most widely used therapeutic agents worldwide. The use of antimicrobials made medical procedures such as organ transplant, cancer chemotherapy, and major surgery relatively safe. ${ }^{1}$ The treatment of bacterial infections was made possible by the discovery of antibiotics, antibacterials that work by either killing bacteria or inhibiting their growth. However, the misuse and mishandling of antibiotics and other antimicrobials by patients as well as health care professionals resulted in the emergence and spread of resistance. ${ }^{1,2}$ Microorganisms that develop resistance show decreased sensitivity to antimicrobials, rendering them ineffective in preventing and treating bacterial, fungal, parasitic, and viral infections. Another important concern is the fact that most of those agents are often associated with adverse side-effects on the host, increasing the risk of complications that could sometimes be deadly. ${ }^{3}$ Those issues, along with several others, provoked the attention of researchers towards traditional treatment. Medicinal plants are of particular interest. ${ }^{2,4}$ Plant oils and extracts are being extensively tested for their potential use as alternative and safe remedies for the treatment of many infectious diseases ${ }^{5,6}$ Turmeric, also known as curcumin is also an example. Turmeric is scientifically called Curcuma longa, a plant that belongs to the Zingiberaceae family. ${ }^{2,7,8}$ Its rhizomes are thick and fleshy, elongated in shape and surrounded by a rough segmented skin. It has a yellowish-brown color with a pale orange color on the inside. ${ }^{3,9}$ The length of the root varies from $2.5 \mathrm{~cm}$ to $7 \mathrm{~cm}$, and the diameter of the main root is generally around $2.5 \mathrm{~cm}$. The plant is native to Southern Asia, and is widely cultivated in the warm areas of the world namely in India, as well as other countries including China, Taiwan, Sri Lanka, Peru and Australia. ${ }^{7,11}$ C. longa is used for several purposes in medicine, cosmetics, food flavoring and textile industries. ${ }^{4,7}$ It has been shown to exhibit major pharmacological activities which include antimicrobial, hepatoprotective, antioxidant, antitumor, wound healing and antiviral effects. ${ }^{4,7,8}$ Furthermore, it displays important therapeutic effects against cardiovascular, pulmonary, metabolic, neurodegenerative and autoimmune diseases. ${ }^{2,10}$ The plant is also prescribed for the treatment of a wide range of health issues related to the digestive tract. ${ }^{11}$ Curcumin has also shown significant antibacterial activity against several strains of both Gram positive and Gram-negative bacteria. ${ }^{9}$ Staphylococcus aureus is one of the Gram-positive strains that was reported to be susceptible to curcuma. ${ }^{9}$ Staphylococcus aureus is a common cause of severe infections in health facilities (nosocomial infections) and the community. ${ }^{3}$ It can cause various infections including infective endocarditis (IE), bacteremia, skin and soft tissue, osteoarticular, and pleuropulmonary infections. ${ }^{9}$ Over the years, S. aureus has evolved and developed multiple strategies to evade the human immune system and to resist antibiotic treatment (MRSA). ${ }^{9}$ The aqueous extract of Curcuma longa has shown antibacterial activity against $S$. aureus. ${ }^{2,11}$ The methanol extract of turmeric also revealed an inhibitory effect against $S$. aureus. ${ }^{3,9}$ The study of hexane and ethanol turmeric extract showed antibacterial activity against 13 bacterial species including $S$. aureus. ${ }^{9}$ Antimicrobial susceptibility tests of different fractions of $C$. longa rhizome extract against $S$. aureus clinical isolates showed that all fractions of $C$. longa rhizome are highly active against standard and clinical isolates of $S$. aureus showing zone of inhibition ranges between $9 \mathrm{~mm}$ and $21 \mathrm{~mm}$. Further observations revealed that the benzene extract was least active, showing zone of inhibition of about $9 \mathrm{~mm}$ at the concentration of $50 \mathrm{mg} / \mathrm{ml}$ while methanolic extract was most active showing zone of inhibition of about $19 \mathrm{~mm}$ at the concentration of $50 \mathrm{mg} / \mathrm{ml}^{3}$. Curcumin was also reported to exhibit an inhibitory effect on methicillin-resistant $S$. aureus. ${ }^{9}$ The purpose of this study was to test the effect of different concentrations of methanol extract of curcumin on local clinical multiresistant strains of S. aureus.

\section{Methods}

\section{Plant used}

Samples of Curcuma longa were purchased from a local farmer's shop, definitively identified by our reference botanist and then immediately sent to the microbiology laboratory for processing. 


\section{Bacterial isolates}

The bacterial isolates used in the study were three multiresistant clinical isolates of Staphylococcus aureus, provided by the Clinical Microbiology Laboratory of the Lebanese American University Medical Center- Rizk Hospital (LAUMC - RH). Randomly, the isolates were named: Staphylococcus aureus isolates 1, 2 and 3. Gram staining and standard biochemical methods were performed to confirm the identity of the test organisms. ${ }^{12}$

\section{Preparation of the methanol extracts}

Two hundred grams of Curcuma longa roots were weighed using a mass balance, chopped and mixed with $80 \%$ methanol in a sterile blender. The extract was then transferred to several Erlenmeyer flasks and kept in an orbital shaker for 1 week. The extracts were then filtered using vacuum assisted Millipore filtration.

\section{Disc agar diffusion}

A volume of $50 \mu \mathrm{L}$ of the extract was added to standard and sterile filter paper discs to be used in the experiment. The Muller Hinton Agar (MHA) plates which were prepared as recommended, ${ }^{13}$ where seeded with an inoculum of the tested strains, that was adjusted to have a turbidity equivalent to a $0.5 \mathrm{McF}$ arland standard. Four of the prepared discs were inserted properly on the surface of each of the seeded plates, after which the plates were incubated at $35^{\circ} \mathrm{C}$ for 24 hours. Any zone of inhibition of growth, around any of the discs, was were then measured using a caliper. The reported results were the average of the diameters measured of the zones of inhibition of growth around the discs inserted on each plate.

\section{Well agar diffusion}

Muller Hinton Agar (MHA) was prepared as recommended. ${ }^{13}$ Using a cork borer wells were introduced in the middle of the MHA plates that were seeded with the test organisms (adjusted to have a

Table I Diameters, in millimeters, of the zones of inhibition of growth of the three Staphylococcus aureus isolates, using different concentrations of the methanol extract of C. longa. C: Control with only methanol*; concentration at which few and scattered resistant colonies observed within the zones of inhibition

\begin{tabular}{|c|c|c|c|c|c|c|c|c|c|c|c|}
\hline & C & $50 \mu \mathrm{L}$ & $100 \mu \mathrm{L}$ & $150 \mu L$ & $300 \mu \mathrm{L}$ & $350 \mu \mathrm{L}$ & $400 \mu \mathrm{L}$ & $450 \mu \mathrm{L}$ & $500 \mu \mathrm{L}$ & $550 \mu \mathrm{L}$ & $600 \mu \mathrm{L}$ \\
\hline $\begin{array}{l}\text { S. aureus } \\
\text { (isolate I) }\end{array}$ & 0 & $13.7^{*}$ & 0 & $8.0^{*}$ & $22.7^{*}$ & $24.0^{*}$ & $25.1 *$ & $27.4^{*}$ & 29.0 & 31.0 & 34.0 \\
\hline $\begin{array}{l}\text { S. aureus } \\
\text { (isolate 2) }\end{array}$ & 0 & $12.4^{*}$ & 0 & 0 & $27.0 *$ & $28.7^{*}$ & $30.4^{*}$ & $31.7^{*}$ & $35.7^{*}$ & $40.4^{*}$ & 43.7 \\
\hline $\begin{array}{l}\text { S. aureus } \\
\text { (isolate 3) }\end{array}$ & 0 & $10.4^{*}$ & 0 & $9.7^{*}$ & $24.4^{*}$ & $30.3^{*}$ & $38.4^{*}$ & $41.0 *$ & $48.3^{*}$ & 51.0 & 57.7 \\
\hline
\end{tabular}

\section{Discussion}

Whereas a volume of $50 \mu \mathrm{L}$ was enough to show a defined zone of inhibition using the disk agar diffusion method, no inhibition was observed when a volume of $100 \mu \mathrm{L}$ was added in the agar well diffusion method. This is attributed to the fact that the kinetics of diffusion of the antimicrobial agents into the agar from a surface application (disk agar diffusion assay) is different from diffusion through the agar (well agar diffusion assay) when the antimicrobial is placed into a well. ${ }^{14}$ This could also be explained by the dependence of the well diffusion assay on several factors including the thickness of the agar. ${ }^{15}$ In addition to that, the fact that the mixture contained different constituents exhibiting different diffusion rates could also be turbidity equivalent to a 0.5 McFarland standard). The different volumes of extract to be tested were added to these wells, after which the plates were incubated at $35^{\circ} \mathrm{C}$ for 24 hours. The plates were then checked for the appearance of zones of inhibition of growth and a caliper was used to measure their diameters. The reported results were the averages of the diameters measured for each of the zones of inhibition of growth that surrounded the wells for each concentration of the tested extract.

\section{Results}

When the disk diffusion assay was performed with $50 \mu \mathrm{L}$ of $C$. longa extract added on the disk, inhibition of the three different isolates used in this experiment were noted (Table 1). When $100 \mu \mathrm{L}$ of extract was added when performing the well agar diffusion assay, no inhibition zone was detected for any of the Staphylococcus aureus isolates used (Tables 1). However, when the volume was raised to $150 \mu \mathrm{L}$, C. longa extract was able to inhibit Staphylococcus aureus isolates 1 and 3 but not Staphylococcus aureus isolate 2 (Table 1). Moreover, when the volume of extract added increased gradually from $300 \mu \mathrm{L}$ to $600 \mu \mathrm{L}$, inhibition zones were recorded for the three different Staphylococcus aureus isolates. Small colonies of Staphylococcus aureus isolate 1 in the zone of inhibition appeared on the plates where the volume of extract added ranged from $50 \mu \mathrm{L}$ to $450 \mu \mathrm{L}$ (Table 1). However, these colonies disappeared when the volume of extract added reached $500 \mu \mathrm{L}, 550 \mu \mathrm{L}$ and $600 \mu$. Small colonies of Staphylococcus aureus isolate 2 in the zone of inhibition also appeared on the plates where the volume of $C$. longa extract added ranged between $50 \mu \mathrm{L}$ and $550 \mu \mathrm{L}$, but a volume of $600 \mu \mathrm{L}$ was able to inhibit the growth of these small colonies. On the plates of Staphylococcus aureus isolate 3 where the volume of $C$. long $a$ added increased from $50 \mu \mathrm{L}$ to $500 \mu \mathrm{L}$, small colonies appeared in the zone of inhibition. Nonetheless, these small colonies disappeared when the volume of extract added reached $550 \mu \mathrm{L}$ and $600 \mu \mathrm{L}$. an explanation of the results obtained. In order to obtain comparable and reproducible results, the techniques used were standardized, as much as possible, by having the inoculum with a density equivalent to 0.5 McFarland, a thickness of agar of $4 \mathrm{~mm}$ in all used plates, and the use of Muller Hinton Agar as recommended. ${ }^{13,16}$ A clear and defined zone of inhibition was observed starting with the addition of $150 \mu \mathrm{L}$ of C. longa extract. As expected, the diameter of the zone of inhibition increased with increasing the volume added until reaching the largest diameter when a volume of $600 \mu \mathrm{L}$ was added (Table 1). Genome sequencing projects for multiple isolates of a bacterial pathogen are of considerable scientific value because the generated data reveals not only their gene content but also the conservation and variability of genes in different strains of the organism. The diversity of S. aureus 
is mainly due to polymorphisms that occur in genomic islands, which carry many virulence and antibiotic resistance determinants. ${ }^{17}$ These differences were demonstrated by the results which showed that at the same volume of $C$. longa extract added for the three $S$. aureus isolates tested, different diameters of the zone of inhibition were observed indicating that each of the strains possessed certain genetic characteristics that rendered it either more or less susceptible to the antibacterial compound(s) found in the natural extract. These differences also accounted for the fact that different volumes of extract were needed to inhibit the appearance of resistant colonies within the zone of inhibition of growth of the three $S$. aureus isolates used.

$S$. aureus is one of the Gram-positive strains that is susceptible to curcumin-mediated inhibition. ${ }^{18}$ However, over the years, $S$. aureus has evolved and developed multiple strategies to evade the human immune system and to resist antibiotics treatment and is thus recognized as a drug fast organism. ${ }^{19,20}$ One of the forms of resistance of $S$. aureus against $C$. longa was revealed by the appearance of small colonies inside the zones of inhibition using different concentration of the methanol extract of C. longa (Table 1). To confirm that these colonies were indeed resistant varieties of the organism, single colonies were isolated from each of plates in which growth was seen within the zones of inhibition, their identities reconfirmed and they were used to run the set of experiments again. The results confirmed our presumption and these resistant strains were able to grow even at high concentrations of the C. longa extract.

\section{Conclusion}

The results confirm that Curcuma longa has an effective antibacterial activity against Staphylococcus aureus. However, in order to use this characteristic to develop pharmaceuticals, the active antibacterial compound in the extract should be identified and purified. This will be later followed by using proper biological assessment assays, dosage formulations, and clinical studies to establish safety, efficacy and the pharmacokinetic profile of any prospective new drug. These steps are important to evaluate the potential of the extract to cause any harm to the host or to the normal flora. ${ }^{21}$ The fact that Curcuma longa is a natural plant whose extract is often used by people without considerable complications, indicates that it is very promising that it may potentially be used in vivo without dangerous side effects. The possibility of incorporating it in common disinfectants must also be considered.

\section{Acknowledgments}

None.

\section{Conflict of interests}

The authors declare that there is no conflict of interest.

\section{References}

1. WHO Fact sheet: Antimicrobial resistance. Geneva: World Health Organization Media Centre; 2018

2. Chakraborty B, Nath A, Saikia H, et al. Bactericidal activity of selected medicinal plants against multidrug resistant bacterial strains from clinical isolates. Asian Pac J Trop Med. 2014;7(1):S435-S441.

3. Gupta A, Mahajan S, Sharma R, et al. Evaluation of antimicrobial activity of Curcuma longa rhizome extract against Staphylococcus aureus. Biotechnology Res. 2015;6:51-55.
4. Naz S, Jabeen S, Ilyas S, et al. Antibacterial activity of Curcuma longa varieties against different strains of bacteria. Pak J Bot. 2010;42(1):455462.

5. Keyrouz E, Abou Raji El Feghali P, Jaafar M, et al. Malva neglecta: A natural inhibitor of bacterial growth and biofilm formation. Journal of Medicinal Plants Research. 2017;11(24):380-386.

6. Ncube NS, Afolayan AJ, Okoh AI, et al. Assessment techniques of antimicrobial properties of natural compounds of plant origin: current methods and future trends. Afr J Biotechnol. 2008;7(12):1797-1806.

7. Awasthi P, Dixit S. Chemical composition of Curcuma longa leaves and rhizome oil from the plains of Northern India. Journal of Young Pharmacists. 2009;1(4):322-326.

8. Singh S, Panda MK, Subudhi E, et al. Chemical composition of leaf and rhizome oil of an elite genotype Curcuma longa L. from South Eastern Ghats of Orissa. J Pharm Res. 2010;3(7):1630-1633.

9. Teow SY, Liew K, Ali SA, et al. Antibacterial action of curcumin against Staphylococcus aureus: a brief review. Journal of Tropical Medicine. $2016 ; 10$.

10. Aggarwal BB, Harikumar KB. Potential therapeutic effects of curcumin, the anti-inflammatory agent, against neurodegenerative, cardiovascular, pulmonary, metabolic, autoimmune and neoplastic diseases. Int $J$ Biochem Cell Biol. 2009;41(1):40-59.

11. Niamsa N, Sittiwet C. Antimicrobial activity of Curcuma longa aqueous extract. Journal of Pharmacology and Toxicology. 2009; 4(4):173-177.

12. Cowan ST, Steel KL. Cowan and Steel manual for the identification of medical bacteria. Cambridge University Press: London; 1974.

13. CLSI. Clinical Laboratory Standards Institute. Performance Standards for Antimicrobial Susceptibility Testing; Twenty-Fourth Informational Supplement. M100-S24. PA: Clinical Laboratory Standards Institute; 2014.

14. Holder IA, Boyce ST. Agar well diffusion assay testing of bacterial susceptibility to various antimicrobials in concentrations non-toxic for human cells in culture. Burns. 1994;20(5):426-429.

15. Davis WW, Stout TR. Disc Plate Method of Microbiological Antibiotic Assay I. Factors Influencing Variability and Error. Applied Microbiol. 1971;22(4):659-665.

16. EUCAST Discussion Document. Determination of minimum inhibitory concentrations (MICs) of antibacterial agents by broth dilution. Clin Microbiol Infect. 2003;9(8):1-7.

17. Baba T, Bae T, Schneewind O, et al. Genome sequence of Staphylococcus aureus strain Newman and comparative analysis of staphylococcal genomes: polymorphism and evolution of two major pathogenicity islands. J Bacteriol. 2008;190(1):300-310.

18. Tong SYC, Davis JS, Eichenberger E, et al. Staphylococcus aureus infections: epidemiology, pathophysiology, clinical manifestations, and management. Clin Microbiol Rev. 2015;28(3):603-661.

19. Liu GY. Molecular pathogenesis of Staphylococcus aureus infection. Pediatr Res. 2009;65(5):71R-77R.

20. Miller LG, Kaplan SL. Staphylococcus aureus: a community pathogen. Infectious Disease Clinics of North America. 2009;23(1): 35-52.

21. Iwu MW, Duncan AR, Okunji CO, et al. New antimicrobials of plant origin. In: Janick J editor. Perspectives on new crops and new uses. ASHS Press: Alexandria; 1999:457-462. 